\title{
Les motivations des acheteurs de produits issus du commerce équitable : des tendances différentes selon les caractéristiques de l'individu
}

Florence de Ferran

Institut de Gestion

Université de La Rochelle

39 rue de Vaux de Foletier

17000 La Rochelle

France

<florence.de_ferran@univ-Ir.fr>

\begin{abstract}
Résumé
L'objet de cet article est d'évaluer le caractère fragmentaire du marché des produits issus du commerce équitable. Pour ce faire, nous avons estimé le caractère modérateur de variables individuelles sur les motivations à l'achat de cafés issus du commerce équitable. Une étude utilisant les chaînages cognitifs a été menée auprès de 174 acheteurs avérés en grandes et moyennes surfaces (GMS). Une analyse s'appuyant sur des cartes hiérarchiques et sur un modèle d'équations structurelles selon les moindres carrés partiels a permis d'établir une certaine hétérogénéité des motivations évoquées et de la structure des chaînages cognitifs.
\end{abstract}

Mots clés : commerce équitable ; comportement du consommateur ; motivation.

Thèmes : alimentation, consommation, nutrition ; transformation, commercialisation.

\section{Abstract \\ Motivating factors in choosing to buy Fair Trade products: tendencies differ according to the individual}

In this paper we examine the fragmented nature of the fair trade products market by assessing the moderating character of individual variables on coffee purchasing motives. A study using a laddering methodology has been implemented among 174 fair trade coffee buyers recruited in supermarkets. An analysis based on hierarchical value maps and a PLS structural equation model makes it possible to identify a relative diversity of motives and means-end structural chains.

Key words: consumer behaviour; fair trade; motivation.

Subjects: consumption, food, nutrition; processing, marketing.
C es dernières années, la médiatisation croissante des problèmes environnementaux et de la responsabilité de l'homme dans leurs amplifications a amené à une prise de conscience de plus en plus forte de ces enjeux. Dans la sphère de la consommation, cela s'est traduit chez certains consommateurs par une évolution des comportements vers plus de frugalité - comportement de simplicité volontaire - ou par des comportements plus responsables. C'est ainsi que le marché des produits porteurs d'une plus grande responsabilité sociale et/ou environnementale s'est développé. Parmi ces produits, nous allons nous intéresser aux produits issus du commerce équitable (désigné dans cet article par la métonymie "produit équitable "), dont la vocation est d'aider les petits producteurs des pays du Sud à vivre dignement de leurs productions et de leur permettre de se développer en les rétribuant plus équitablement. Ces produits connaissent aujourd'hui un engouement important avec un marché de 2,4 milliards d'euros à l'échelle mondiale - dont 256 millions d'euros en France - et un taux de croissance à deux chiffres depuis de nombreuses années ${ }^{1}$.

Parallèlement, les recherches en marketing ont témoigné d'un intérêt croissant pour l'étude des déclencheurs de cet achat.

\footnotetext{
Ressources Max Havelaar: http://www. maxhavelaarfrance.org/En-chiffres.
} 
Toutefois, la majorité d'entre elles n'ont pas considéré l'acheteur de produits équitables comme un individu protéiforme, alors que l'on assiste à une fragmentation des marchés (Lipovetsky et Charles, 2004) et que les travaux de Ozcaglar (2006) et de De Ferran et Grunert (2007) ont mis en évidence des mobiles différents à l'achat de cette catégorie de produits. On peut alors s'interroger sur la nature des variables qui déterminent cette diversité de motivations à travers l'étude des facteurs internes et externes à l'individu, facteurs qui conditionnent son comportement (Ward et Robertson, 1973, in Belk, 1975).

Cette connaissance est importante tant sur un plan théorique, afin d'approfondir la connaissance de l'acheteur de produits équitables, que sur un plan " managérial ", afin de mettre en ouvre des stratégies de segmentation différenciées au fur et à mesure que se développe ce marché.

\section{Déterminants de l'achat de produits équitables}

Quelques recherches ont permis de qualifier plus précisément l'acheteur de produits équitables, que ce soit à travers la définition de typologies de consommateurs (De Pelsmacker et al., 2004 ; Sirieix et al., 2004 ; Ozcaglar, 2006 ; De Ferran et Grunert, 2007) ou celle de facteurs déterminant cet achat (Shaw et Shui, 2001 ; Ozcaglar-Toulouse et al., 2006). Il apparaît comme un individu idéaliste, sensible aux déséquilibres entre les pays du Nord et les pays du Sud. Il estime que son achat permet d'agir sur le système et ressent une obligation morale de le faire. Il s'agit plutôt de femmes, d'âge moyen et ayant un haut niveau d'études, qui acceptent de payer un supplément pour ces produits et qui achètent aussi des produits issus de l'agriculture biologique.

Quant aux recherches qui ont tenté d'expliquer ce comportement par l'application du modèle de l'action planifiée, elles ont abouti à un pouvoir explicatif faible dans un contexte français - le coefficient de détermination $\mathrm{R}^{2}$ variant de 0,12 à 0,19 - et ce même après l'introduction de variables complémentaires comme l'image de soi et l'obligation éthique $-\mathrm{R}^{2}$ variant de 0,15 à 0,29 (Ozcaglar-Toulouse et al., 2005; Ozcaglar-Toulouse et al., 2006).
Au-delà des limites méthodologiques de ces recherches - échantillon de très petite taille, mesure d'intention d'achat pour caractériser l'acheteur - la majorité d'entre elles ont étudié cet achat comme un comportement homogène. Or, au moins deux recherches nous amènent à penser le contraire :

- d'une part, Ozcaglar-Toulouse et al. (2006) présentent des processus de choix différents selon la fréquence d'achat des produits équitables. L'intention d'achat de ces produits est alors fonction des attitudes et des normes subjectives, c'est-à-dire des normes imposées par l'entourage pour ceux qui en achètent peu ou pas. En revanche, elle est fonction des attitudes et du contrôle comportemental perçu, c'est-à-dire de la facilité ou de la difficulté perçue à effectuer cet achat, pour ceux qui achètent fréquemment ces produits.

- d'autre part, De Ferran et Grunert (2007) montrent que les motivations à l'achat du café équitable sont différentes selon le point de vente fréquenté : les acheteurs en GMS font cet achat surtout par hédonisme, puis pour le bien-être des petits producteurs, alors que les acheteurs en magasins spécialisés sont tout autant motivés par une volonté d'égalité entre les hommes et de préservation de l'environnement, que par une satisfaction personnelle liée à la consommation d'un bon produit.

Des facteurs externes et internes à l'acheteur conduiraient à des processus de choix différents. Le comportement d'achat de produits équitables semble donc être un comportement hétérogène qu'il convient de mieux définir par l'identification de ces facteurs.

\section{Facteurs caractérisant des processus
de choix différents}

De nombreux facteurs peuvent être à l'origine de processus de choix différents. Nous avons choisi de nous centrer sur les caractéristiques de l'acheteur car elles ont une influence sur les bénéfices perçus, sur la valeur attribuée à un produit (Lai, 1995) et, donc, sur les éléments qui motivent le choix. Nous avons considéré des variables sociodémographiques et comportementales selon leur impact sur les motivations qui conduisent un comportement ou selon leur impact sur d'autres comportements d'achat socialement responsables comme l'achat de produits issus de l'agriculture biologique.

\section{Variables sociodémographiques}

Si l'on s'intéresse aux valeurs qui témoignent de la nature des motivations poursuivies, de nombreuses recherches attestent du rôle modérateur du genre, de l'âge et de la profession.

Pour ce qui est du genre, les différences de valeurs observées entre les hommes et les femmes s'expliqueraient par l'apprentissage de rôles distincts lors de la socialisation (Rokeach, 1973) : les hommes sont plus hédonistes, orientés vers la réussite et matérialistes, alors que les femmes sont plus conduites par le bonheur intérieur, l'amour, le respect d'elles-mêmes et l'absence de conflit. Ces différences s'expliqueraient aussi par le fait que les hommes et les femmes possèdent à leur naissance des dispositions distinctes, qu'ils n'ont pas apprises, autrement dit des instincts propres. Des études utilisant la typologie des valeurs de Rokeach attestent également d'une orientation des valeurs différentes selon le genre (Feather, 1984). Quant au caractère social de la profession, c'est-à-dire toute activité professionnelle liée à l'éducation, la santé et à l'action sociale, il serait un indicateur des valeurs de l'individu. En ce sens, les étudiants en médecine témoignent d'une plus grande importance des valeurs à caractère social telles que l'altruisme par rapport aux étudiants en psychologie, sachant que le choix de devenir médecin est souvent fonction du souhait d'aider les autres (Feather, 1982). Ainsi, un individu exerçant une profession à caractère social présenterait une plus grande orientation sociale de ses valeurs par rapport à un individu qui n'exercerait pas une profession à caractère social.

Quant à l'âge, il apparaît comme une variable discriminante des structures cognitives sous-jacentes à des comportements d'achat socialement responsables. Dans le cadre de l'achat de produits verts, les moins de 30 ans sont motivés par le respect de l'environnement et sont tournés vers le futur, tandis que les plus de 40 ans sont ancrés dans le présent, veulent éviter le gaspillage et profiter de ce qu'ils possèdent (Kréziak, 1998). Il en est de même pour l'achat de produits biologiques : les individus de 41 à 50 ans privilégient la sécurité 
des aliments, alors que les plus jeunes privilégient le goût et la préservation de l'environnement (McEachern et McClean, 2002).

\section{Variables comportementales}

Nous avons considéré la pratique de comportements socialement responsables, car leur adoption conduit à la mise en œuvre d'autres comportements de cette nature si les valeurs de l'individu sont orientées socialement (Thøgersen et Ölander, 2001). Ainsi, un individu qui a plusieurs comportements socialement responsables aura un système de valeurs différent des individus qui n'ont pas ces mêmes comportements. Ces comportements étant nombreux, nous nous sommes limités à l'engagement dans l'achat de produits équitables, la pratique de comportements militants et de comportements de dons.

L'engagement dans l'achat d'un produit est une motivation intérieure à l'adoption et à la diffusion de ce produit (Kotler et Simon-Miller, 1984). Il témoigne donc du degré d'investissement de l'acheteur par rapport à un produit, ce qui devrait se traduire par des processus de choix différents. Ozcaglar-Toulouse et al. (2006) le montrent, pour une part, avec des processus d'achat différents selon la fréquence d'achat de produits équitables. Il en est de même pour l'achat de produits biologiques dont les motivations sont fonction de la fréquence d'achat (Zanoli et Naspetti, 2002).

Par ailleurs, la pratique de comportements militants et de comportements de don a une influence sur les comportements d'achat socialement responsables. L'engagement dans des activités militantes ou politiques visant à combattre la dégradation de l'environnement - telles que l'écriture dans des journaux et/ou la participation à des groupes de pression - a une influence positive sur l'achat de détergents écologiques ou de fruits et légumes biologiques (Schlegelmilch et al., 1996). Les déterminants à l'achat de produits équitables devraient donc différer selon la pratique de comportements militants.

Quant aux comportements de don, ils peuvent être définis en dons de temps et en dons d'argent (Schlegelmilch et Tynan, 1989), le don de temps étant associé au bénévolat, et le don d'argent, à des dons pécuniaires à des associations. Là aussi, leurs pratiques témoigneraient d'une certaine orientation des valeurs de l'individu.

\section{Méthodologie}

\section{Une approche par les chaînages cognitifs}

Afin d'estimer l'hétérogénéité des motivations à l'achat de produits équitables, nous nous sommes fondés sur une analyse des perceptions de l'acheteur qui peut s'affranchir des perceptions du chercheur. La théorie des chaînages cognitifs a été retenue comme une théorie et une méthodologie centrale de notre recherche, car elle permet de déterminer les motivations et, plus particulièrement, la place des valeurs dans le jugement à travers le choix des attributs du produit. Issue de la psychologie des construits personnels de Kelly (1955), cette théorie a été adaptée par Reynolds et Gutman (1988) à l'étude du comportement du consommateur afin de comprendre les structures cognitives associées à l'achat d'un produit, c'està-dire les relations entre les attributs du produit, les bénéfices qu'en retire le consommateur, et les liens éventuels avec ses valeurs personnelles. Ces structures prennent la forme suivante :

attributs $\rightarrow$ conséquences $\rightarrow$ valeurs.

Les chaînages cognitifs relatifs à l'achat de produits équitables ont donc été définis et relativisés au regard de variables modératrices que sont les variables sociodémographiques et comportementales présentées. Dans ce cadre, nous avons répondu à deux questions :

- est-ce que la nature des éléments constitutifs des chaînages cognitifs varie? c'est-à-dire : est-ce que les attributs, les bénéfices et les valeurs, changent selon chacune des variables individuelles étudiées?

- est-ce que la forme des chaînages cognitifs varie? C'est-à-dire : est-ce que le pouvoir explicatif et la hiérarchie des éléments déterminants du choix diffèrent selon chacune des variables individuelles étudiées?

\section{Échantillon}

Cette recherche a été menée auprès de 174 acheteurs de café équitable, car ce produit est celui qui possède la plus forte part de marché. Les répondants ont été recrutés au moment de leur achat, afin qu'on soit sûr de s'adresser à des acheteurs avérés, et dans un seul canal de distribution que sont les GMS, pour une plus grande homogénéité des résultats.

\section{Collecte des données et méthodes d'analyses}

Les chaînages cognitifs ont été collectés par la méthode des protocoles écrits avec un support (de Ferran, 2006). Elle consiste, dans une première phase, à générer l'ensemble des attributs, conséquences et valeurs par l'intermédiaire d'entretiens individuels, puis, dans une seconde phase, à générer l'ensemble des chaînages cognitifs relatifs à l'achat de café équitable via la technique des protocoles écrits. Cette seconde phase a conduit à la définition de chaînages selon quatre niveaux : 1) les attributs ; 2) les conséquences ; 3) les valeurs instrumentales, qui correspondent à un mode de comportement et qui sont un instrument pour atteindre 4) les valeurs terminales, considérées comme les buts de l'existence.

Vingt acheteurs ont été interrogés dans la première phase de collecte des chaînages, afin de formaliser le support nécessaire à la seconde phase, qui a été menée auprès de 154 acheteurs et qui a permis de collecter 580 chaînages cognitifs, soit 3,8 chaînages par répondant.

Quant aux variables individuelles, elles ont été collectées par le biais d'un questionnaire autoadministré. Les échelles de mesures des variables comportementales sont présentées en annexe 1.

Les données ainsi collectées ont été analysées par différentes méthodes :

- des cartes hiérarchiques afin de déterminer la nature des motivations poursuivies ; - un modèle d'équations structurelles selon les moindres carrés partiels (PLS) et des tests de comparaison des moyennes afin de définir les variables qui conditionnent le choix.

Ces analyses ont été conduites pour chacune des variables individuelles considérées et sur deux groupes d'individus de manière à établir le caractère modérateur de la variable. Ces groupes ont été formalisés sous la contrainte des caractéristiques de nos répondants et de manière à comparer deux ensembles de taille proche.

\section{Résultats}

\section{Variabilité de la nature des éléments composant les chaînages cognitifs}

Les chaînages cognitifs des 25-35 ans (versus ceux des plus de 35 ans), des hommes (versus ceux des femmes), et des acheteurs 
ayant une profession à caractère social (versus ceux qui n'ont pas une profession à caractère social) sont proches avec des coefficients de corrélation élevés. Toutefois, ils ne sont pas identiques et présentent des spécificités aux niveaux :

- des éléments cités :

- les plus jeunes accordent de l'importance au label équitable et au fait de se comporter de manière réfléchie, contrairement au plus de 35 ans qui ont évoqué le respect des droits de l'homme;

- les hommes accordent de l'importance à des éléments concrets tels que la qualité et le label qui permet une traçabilité, alors que les femmes l'accordent à des éléments sociaux transcendants tels que le respect des droits de l'bomme que permet l'achat d'un produit équitable auquel des petits producteurs ont participé ;

- quant à ceux qui exercent une profession à caractère social, ils accordent plus d'importance au fait d'être en harmonie avec eux-mêmes, alors que les autres évoquent le fait de se comporter de manière réfléchie, ainsi que le respect des droits de l'bomme;

- des dimensions principales des cartes hiérarchiques (figures 1 et 2)

- bien que l'ensemble des acheteurs soient principalement conduits par des motivations de nature individuelle comme la satisfaction, les acheteurs de plus de 35 ans ou de sexe féminin, accordent plus d'importance au caractère biologique du produit et au respect de l'environnement qui en résulte, qu'au caractère équitable qui est plus important pour les 25-35 ans et pour les hommes ;

- ces derniers attachent également plus d'importance au fait d'apporter une contribution durable à la Société que les plus âgés qui sont moins idéalistes. Les hommes sont alors autant motivés par la satisfaction que par la contribution durable que peut apporter l'achat de café équitable, alors que les femmes accordent plus d'importance à la qualité du produit, au fait qu'il soit bon et qu'il soit biologique ;

- quant aux acheteurs qui exercent une profession à caractère social, ils accordent plus d'importance au caractère équitable du produit alors que les autres privilégient son caractère biologique ;

- de la hiérarchie des éléments les plus centraux :

- au regard des coefficients de centralité (Pieters et al., 1995), le caractère responsable est plus central pour les acheteurs qui ont entre 25 et 35 ans, alors qu'il s'agit du caractère juste pour les plus de 35 ans ;

- quant aux acheteurs qui ont une profession à caractère social, l'égalité entre les bommes et la participation à une économie alternative sont plus centrales que la satisfaction, la qualité du produit et son caractère biologique, qui sont plus centraux pour les acheteurs qui n'ont pas une profession à caractère social ;

- de la complexité des structures cognitives, plus forte pour les acheteurs de plus de 35 ans, mais aussi pour les femmes au niveau des relations entre les valeurs et les conséquences, et pour les acheteurs qui n'exercent pas une profession à caractère social.

On retrouve cette même hétérogénéité de motivations selon les variables comportementales. L'ensemble des spécificités au niveau des éléments cités, de la hiérarchie des éléments les plus centraux, des dimensions principales des cartes hiérarchiques et de la complexité des structures cognitives nous amène à penser que le degré d'engagement de l'acheteur, la pratique de comportements militants et de comportements de don ont une influence sur les éléments composant les chaînages cognitifs à l'achat de café équitable. Les cartes hiérarchiques selon le degré d'engagement dans l'achat de café équitable sont présentées en figures 3 et 4 ; les autres peuvent être communiquées sur demande.

Quant à la nature de ces motivations, on note une prévalence des éléments de nature individualiste et hédoniste, et une importance variable des éléments de nature universaliste ou transcendantale. Ainsi, les acheteurs qui sont faiblement engagés dans cet achat, qui ne sont pas militants ou qui donnent de leur temps et de leur argent, sont davantage motivés par des éléments de nature individuelle comme la satisfaction, alors que les acheteurs qui sont fortement engagés vis-à-vis de cet achat, qui sont militants, qui ne donnent pas de leur temps ou de leur argent sont autant conduits par des motivations de nature sociale qu'individuelle. Les acheteurs de café équitable poursuivent donc des motivations différentes selon les variables individuelles étudiées.

\section{Variabilité de la structure des chaînages cognitifs}

La mise en œuvre d'un modèle PLS sur chacun des deux sous-échantillons associés à une même variable conduit à l'obtention de la structure la plus représen- tée des chaînages cognitifs qui est la première dimension extraite par l'analyse. Les mêmes relations structurelles les plus importantes se retrouvent dans les deux groupes pour chacune des variables étudiées, à savoir : les valeurs terminales déterminent le plus largement les conséquences qui, elles-mêmes, déterminent le plus les attributs importants du choix. Cependant, si l'on considère l'ensemble des relations structurelles, nos résultats témoignent de structures différentes selon l'âge, le caractère social de la profession, le degré d'engagement dans l'achat et la pratique de comportements militants, car l'ensemble des tests d'égalité des coefficients de dépendance attestent de différences significatives à un seuil de risque de $5 \%$ et certaines relations structurelles ne sont pas significatives. Ainsi, nous avons déterminé trois structures de chaînages cognitifs selon les variables étudiées. Elles sont présentées dans le tableau 1 avec les variables qui les caractérisent.

Les deux premières structures présentées dans le tableau 1 sont les plus récurrentes. Elles se distinguent par l'existence, ou non, d'une relation causale entre les valeurs terminales et les attributs du produit, qui confère un caractère plus ou moins symbolique à l'acte d'achat.

Un acheteur de café équitable qui n'exerce pas une profession à caractère social, qui n'a pas de comportement militant, ou qui est fortement engagé dans cet achat, est susceptible de choisir le café équitable selon les bénéfices apportés par le produit et les valeurs terminales qu'il pourra exprimer à travers cet achat. Les valeurs conditionnent donc, pour une part, le comportement de manière directe, même si cette part reste faible.

Quant au processus de choix décrit par la deuxième structure, il est plus utilitaire, puisque l'achat est motivé par les bénéfices apportés par le produit, qui seront eux-mêmes définis par les valeurs sousjacentes à l'achat. Le jugement associé à cet achat est alors plus fragmentaire.

Le profil de l'acheteur a donc une influence sur le processus de choix associé à l'achat de café équitable.

Pour ce qui est du genre et de la pratique de dons, ces variables n'induisent qu'une variation de l'intensité des relations, tout en conservant une structure des chaînages identique. Le processus de choix est alors fonction des mêmes éléments, bien que l'intensité de leur influence soit différente. 


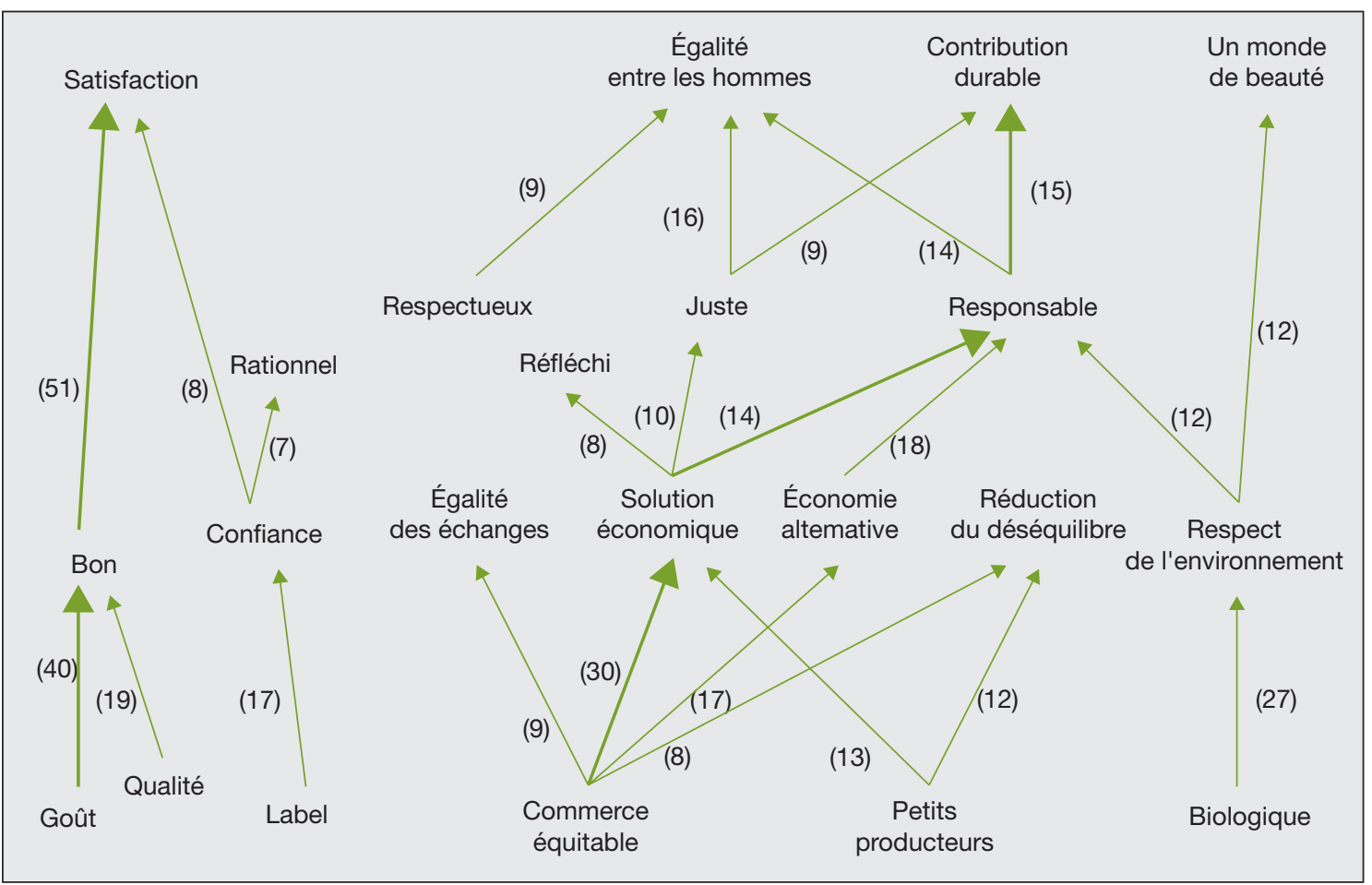

Figure 1. Carte hiérarchique de l'achat de café équitable par les $25-34$ ans ( $n=68$; $n_{\text {chaînage }}=255$; seuil = 7).

Figure 1. Hierarchical map of Fair Trade coffee purchases by $25-34$ year-olds $\left(n=68 / n_{\text {linking }}=255 /\right.$ threshold $=7$ ).

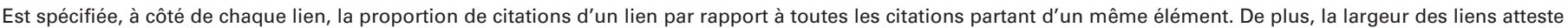
de leur importance.

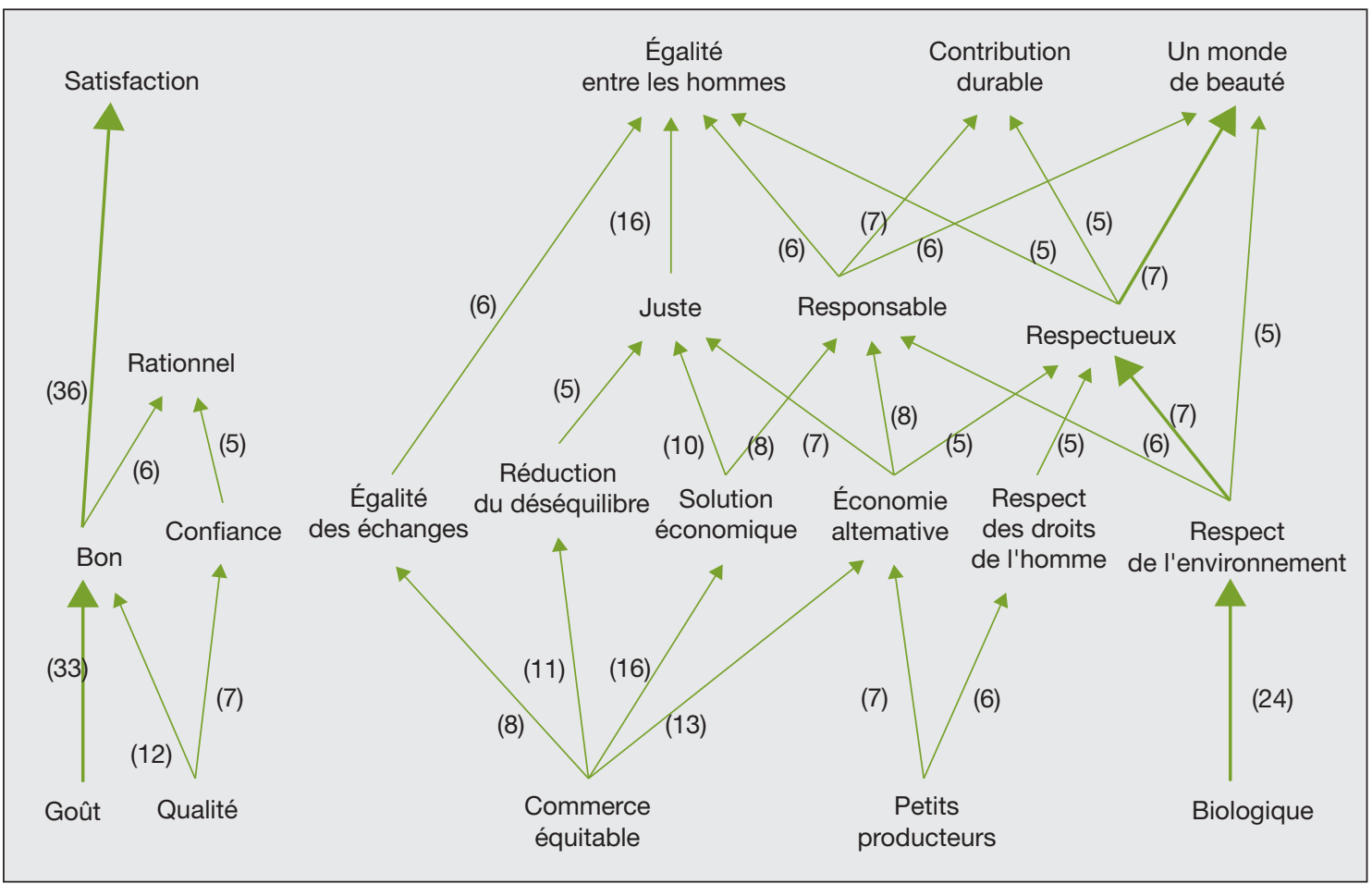

Figure 2. Carte hiérarchique de l'achat de café équitable par les 35 ans et plus $\left(n=68 ; n_{\text {chaînage }}=255\right.$; seuil $=7$ ).

Figure 2. Hierarchical map of Fair Trade coffee purchases by 35 year-olds and older $\left(n=51 / n_{\text {linking }}=190 /\right.$ threshold $=5$ ).

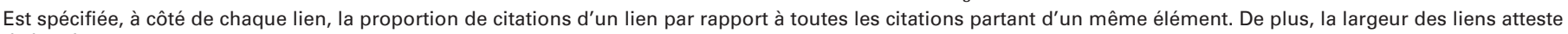
de leur importance. 


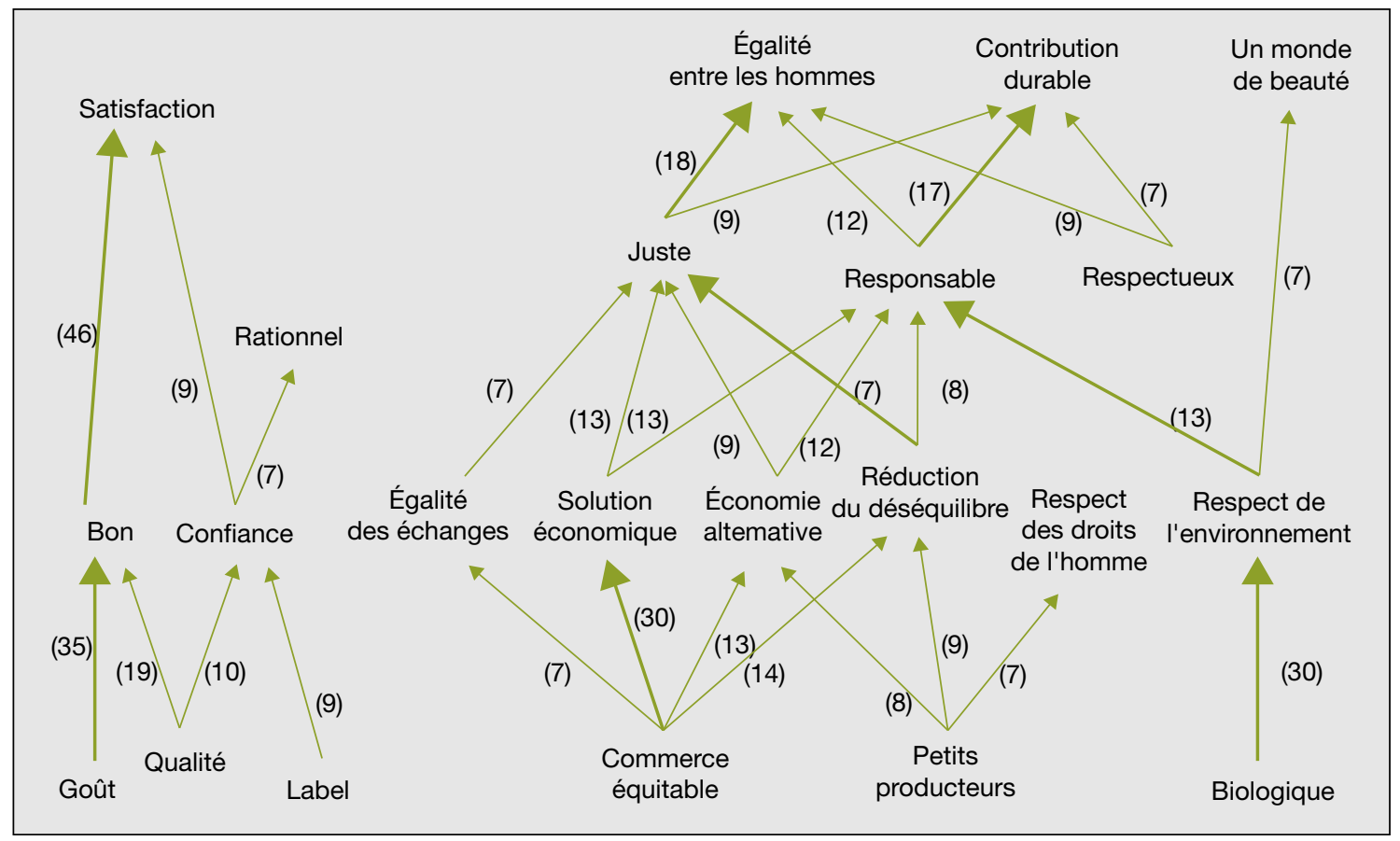

Figure 3. Carte hiérarchique de l'achat de café équitable par les acheteurs fortement engagés dans cet achat $\left(n=68 ; n_{\text {chaînage }}=255 ;\right.$ seuil $\left.=7\right)$.

Figure 3. Hierarchical map of Fair Trade coffee purchases by buyers closely attached to such purchases $\left(n=66 / n_{\text {linking }}=255 /\right.$ threshold $=7$ ). Est spécifiée, à côté de chaque lien, la proportion de citations d'un lien par rapport à toutes les citations partant d'un même élément. De plus, la largeur des liens atteste de leur importance.

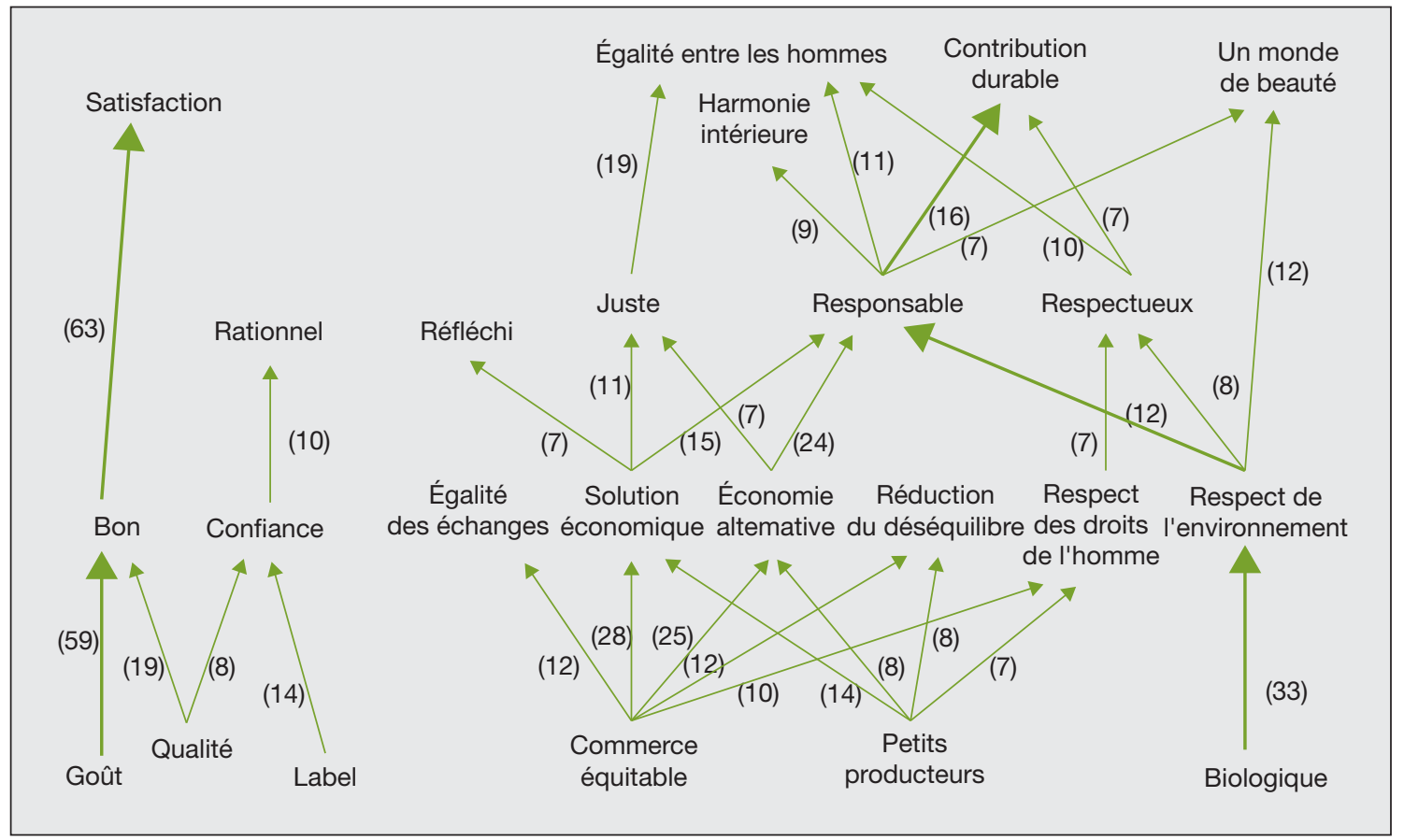

Figure 4. Carte hiérarchique de l'achat de café équitable par les acheteurs moyennement ou peu engagés dans cet achat $\left(n=68 ; n_{\text {chaînage }}=255 ;\right.$ seuil $\left.=7\right)$.

Figure 4. Hierarchical map of Fair Trade coffee purchases by buyers moderately or only weakly attached to such purchases $\left(n=88 / n_{\text {linking }}=325 /\right.$ threshold $\left.=7\right)$. Est spécifiée, à coté de chaque lien, la proportion de citations d'un lien par rapport à toutes les citations partant d'un même élément. De plus, la largeur des liens atteste de leur importance. 


\section{Tableau 1. Les différentes structures des chaînages cognitifs à l'achat de café équitable en GMS selon les variables sociodémographiques et comportementales étudiées.}

Table 1. The different structures of the cognitive chains involved in choosing to buy Fair Trade coffee in supermarkets according to the socio-demographic and behavioural variables studied.

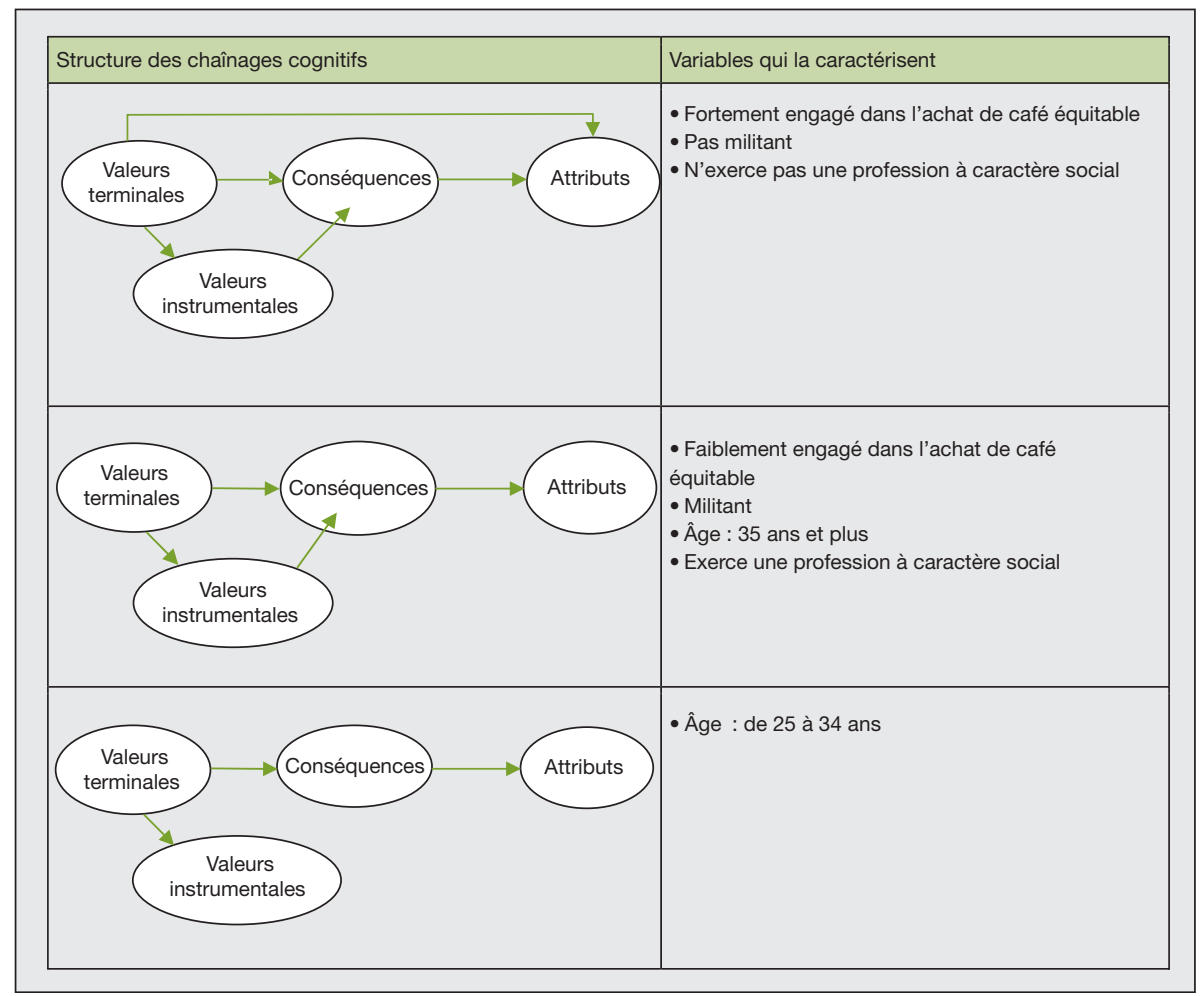

\section{Discussion}

\section{et conclusion}

Les chaînages cognitifs sous-jacents à l'achat de café équitable présentent des contenus et des structures différentes selon les variables sociodémographiques et comportementales étudiées. Toutefois, cette diversité des motivations est relative, car il existe des correspondances assez importantes au niveau des cartes hiérarchiques. Ainsi, l'achat de café équitable est motivé par des éléments de nature individuelle, quelles que soient les caractéristiques de l'acheteur, et, à des degrés divers, par des éléments de nature universaliste et/ou transcendantale selon le profil de l'acheteur. Tout individu consomme donc pour satisfaire ses besoins primaires quelle que soit la part altruiste de sa consommation (Strong, 1997). Au-delà de ce point commun, conformément à ce qui était attendu, ceux qui ont une profession à caractère social accordent plus d'importance à des motivations de nature sociale, contrairement aux autres qui pri- vilégient la qualité du produit et/ou son caractère biologique dans un objectif de satisfaction. De même, les acheteurs de plus de 35 ans (versus les plus jeunes) privilégient la qualité d'un produit, voir son caractère biologique, avant son caractère équitable. Ils sont donc moins idéalistes et plus portés par des motivations de nature individuelle, ce que l'on retrouve dans la recherche de McEachern et McClean (2002) sur les produits biologiques. Il existe donc une certaine homogénéité entre les motivations à l'achat de produits équitables et celles à l'achat de produits biologiques, ce qui rend cohérent la commercialisation de produits bio-équitables.

En revanche, contrairement aux valeurs attribuées aux hommes et aux femmes, les motivations d'achat des hommes sont moins orientées vers la satisfaction individuelle que celles de femmes. Cela peut s'expliquer par le fait que, malgré l'évolution de la société, les femmes conservent la responsabilité de la majorité des tâches domestiques (Putrevu, 2002); de plus, elles achètent pour elles et/ou pour leur famille alors que les hommes achètent généralement pour eux seuls. Il est donc assez cohérent qu'elles se préoccupent de la satisfaction que leur foyer tirera de la consommation des différents produits achetés, et non d'un engagement particulier qui est un acte individuel.

Concernant les militants et les consommateurs engagés dans cet achat, ils accordent autant d'importance au goût et à la qualité du produit qu'à ses caractères biologique et équitable, alors que ceux qui ne donnent ni de leur temps, ni de leur argent accordent plus d'importance à la dimension sociale de l'achat de café équitable, ce qui est contraire à notre postulat. Ce résultat pourrait s'expliquer par des buts poursuivis différents selon la nature des comportements socialement responsables. Des recherches mériteraient d'être conduites, afin de définir une typologie de comportements socialement responsables selon les buts qu'ils permettraient d'atteindre et selon les caractéristiques des individus.

L'ensemble de ces résultats attestent donc de l'hétérogénéité des motivations à l'achat de café équitable. Le profil de l'acheteur équitable n'est pas unique. Il convient donc d'être vigilant lors de l'utilisation des 
connaissances sur le profil de cet acheteur ou sur les déclencheurs de son comportement d'achat.

Par ailleurs, nos résultats présentent l'achat de café équitable comme relevant davantage d'un jugement fragmentaire et d'un comportement utilitaire. Cet utilitarisme est à nuancer pour ceux qui sont fortement engagés dans cet achat, qui n'exercent pas une profession à caractère social et/ou qui n'ont pas de comportements militants, car il revêt un caractère plus symbolique. Il s'inscrirait alors parfaitement dans une vision hypermoderne de la consommation où l'individu tenterait de conjuguer sa quête de jouissance avec un comportement porteur de sens (Lipovetsky et Charles, 2004).

Sur un plan "managérial ", cette recherche a contribué à une meilleure définition des éléments propres aux stratégies de positionnement et de communication par des critères de segmentation utiles à une communication ciblée et par des éléments sur lesquels communiquer selon le public visé. Par exemple, si l'on souhaite formaliser des communications selon le caractère plus ou moins militant des individus, il serait nécessaire de mettre en avant la dimension engagée de cet achat pour les militants, et le goût, la qualité du produit et son caractère équitable ou biologique pour les autres. En revanche, pour certaines variables étudiées comme l'âge, on constate qu'il n'y a pas de différence fondamentale sur les principaux élément sur lesquels communiquer : les produits équitables sont des produits de qualité qui ont bon goût et qui apportent une solution économique aux problèmes de développement. Toutefois, le caractère biologique du produit qui préserve l'environnement peut être un axe de communication secondaire à destination d'une cible d'individus de plus de 35 ans. Au-delà d'une définition plus précise des motivations à l'achat de produits équitables et de la mise en évidence de leur relative diversité, cette recherche présente des limites au niveau de la validité des résultats obtenus, du fait de l'étude d'un seul produit équitable, le café, et d'acheteurs fréquentant un seul canal de distribution, les GMS. De plus, la méthodologie employée a peut-être surestimé le caractère utilitaire du comportement. D'autres recherches seront donc nécessaires pour une meilleure connaissance des déclencheurs de cet achat et de leur diversité.

\section{Références}

Belk RW. Situational variables and consumer behavior. J Consum Res 1975; 2 : 157-64.

De Ferran F. A comparison of three laddering techniques applied to a socially desirable product, the fair trade coffee. Proceedings of the $35^{\text {th }}$ EMAC Conference, Athens, 2006.

De Ferran F, Grunert K. French fair trade coffee buyers' purchasing motives: An exploratory study using means-end chains analysis. Food Qual Prefer 2007 ; 18 : 218-29.

De Pelsmacker P, Driesen L, Rayp G. Are faire trade labels good business? Ethics and coffee buying intentions. Proceedings of the 33rd EMAC Conference, Murcia, 2004.

Ellen PS, Wiener JL, Cobb-Walgren C. The role of perceived consumer effectiveness in motivating environmentally conscious consumer. J Public Policy Mark 1991 ; 10 : 102-17.

Feather NT. Reasons for entering medical school in relation to value priorities and sex of student. J Occup Psychol 1982 ; 55 : 119-28.

Feather NT. Masculinity, feminity, psychological androgyny, and the structure of values. J Pers Soc Psychol 1984 ; 47 : 604-20.

Heberlein TE, Black JS. Cognitive consistency and environmental action. Environ Behav $1981 ; 13: 717-34$

Kelly GA. The psychology of personal constructs. New York: Norton, 1955.

Kotler P, Simon-Miller FL. Marketing et consommation: À la recherche de l'acheteur perdu. Rev Fr Marketing 1984; 100 : 11-22.

Kréziak D. Les motivations de la consommation verte : une approche par les chaînages cognitifs. Thèse de doctorat, université Pierre Mendès-France, ESA Grenoble, 1998.

Lai AW. Consumer values, product benefits and consumer value: a consumption behavior approach. Adv Consum Res 1995; 22 : 381-7.

Lipovetsky G, Charles S. Les temps hypermodernes. Paris: Grasset, 2004.
McEachern MG, McClean P. Organic purchasing motivations and attitudes: are they ethical ? International Journal of Consumer Studies $2002 ; 26$ : 85-92.

Ozcaglar N. Apport du concept d'identité à la compréhension du comportement du consommateur responsable : une application à la consommation de produits issus du commerce équitable. Thèse de doctorat, université Lille 2, ESA, 2006.

Ozcaglar-Toulouse N, Shiu E, Shaw D. In Search of fair trade: ethical consumer decision-making in France. International Journal of Consumer Studies 2006 ; 30 : 502-14.

Ozcaglar-Toulouse N, Hassan L, Shui E, Shaw D. Understanding ethical consumer decision-making: comparing and contrasting France and the UK. Actes du congrès annuel de I'Academy of Marketing, Dublin, 2005.

Pieters R, Baumgartner $\mathrm{H}$, Allen D. A meansend chain approach to consumer goal structures. Int J Res Mark 1995; 12 : 227-44.

Putrevu S. Exploring the origins and information processing differences between men and women: implications for advertisers. Academy of Marketing Science Review $2002 ; 6$ : 1.

Reynolds TJ, Gutman J. Laddering theory, method, analysis, and interpretation. J Advertising Res $1988 ; 28: 11-31$.

Rokeach M. The nature of human values. New York: The Free Press, 1973.

Schlegelmilch BB, Tynan C. Who volunteers? An investigation into the characteristics of charity volunteers. Journal of Marketing Management $1989 ; 5: 133-51$

Schlegelmilch BB, Bohlen GM, Diamantopoulos $A$. The link between green purchasing decisions and measures of environmental consciousness. Eur J Marketing 1996; 30 : 35-55.

Shaw D, Shui E. Ethics in consumer choice: a multivariate modelling approach. Eur J Marketing $2001 ; 37$ : 1485-98.

Sirieix L, Meunier A, Schaer B. Les consommateurs et le commerce équitable : scepticisme, confiance accordée et disposition à s'engager. Economie et Sociétés 2004 ; 38 : 571-90.

Strong C. The problems of translating fair trade principles into consumer purchase behavior. Marketing Intelligence \& Planning 1997 $15: 32-7$.

Thøgersen J, Ölander F. Spillover of environment-friendly consumer behaviour. Proceedings of the 5th Nordic Environment Research Conference, Aarhus, 14-16 June, 2001.

Zanoli R, Naspetti S. Consumer motivation in the purchase of organic food. Brit Food $J$ $2002 ; 104: 643-53$. 


\section{Annexe 1 \\ Instruments de mesures}

Engagement à l'achat de produits équitables (dimension subjective de l'engagement (Heberlein et Black, 1981))

- Depuis combien de temps achetez-vous du café issu du commerce équitable?

Moins d'1 an/ 1 à 2 ans/ 2 à 3 ans/ 3 ans et plus

- Sur vos cinq derniers achats de café, combien étaient des achats de café issu du commerce équitable?

.../cinq achats de café

Comportements militants (Ellen et al., 1991 ; Schlegelmich et al., 1996)

- Actuellement, est-ce que vous signez des pétitions pour des causes humanitaires ou sociales?

- Actuellement, est-ce que vous êtes membre d'un groupe de pression (groupe qui défend une cause à caractère social, économique, environnemental (ex : ATTAC)?

\section{Oui/non}

\section{Si oui :}

- Est-ce que vous participez à des évènements ou des activités d'un groupe de pression (manifestation, forum...)?

- Est-ce que vous soutenez financièrement un ou plusieurs groupes de pression?

Comportements de don (Schlegelmilch et Tynan, 1989)

- Actuellement, est-ce que vous êtes bénévole (dans une association ou autres)?

Oui/non

- Actuellement, est-ce que vous donnez de l'argent à des causes humanitaires ou sociales?

Toutes les mesures ont été effectuées sur des échelles de Likert en cinq points sauf mention contraire en italique 